\title{
A Study of Differential Gene Expression and Core Canonical Pathways Involved in Rhenium Ligand Treated Epithelial Mesenchymal Transition (EMT) Induced A549 Lung Cancer Cell Lines by INGENUITY Software System
}

\author{
Christopher Krauss ${ }^{1,2}$, Chelsey Aurelus' ${ }^{1}$, Kayla Johnston'1, Joseph Hedley1, Satyendra Banerjee1, \\ Sarah Wisniewski', Quentin Reaves', Khadimou Dia' ${ }^{1}$, Shenell Brown'1, Victoria Bartlet ${ }^{1}$, \\ Sheritta Gavin ${ }^{1}$, Jazmine Cuffee ${ }^{1}$, Narendra Banerjee', Kuldeep Rawat ${ }^{1}$, Santosh Mandal' \\ Zahidur Abedin ${ }^{3}$, Somiranjan Ghosh ${ }^{4}$, Hirendra Banerjee ${ }^{*}$
}

${ }^{1}$ Department of Natural Sciences and Department of Health and Human Studies, Elizabeth City State University, University of NC, Elizabeth City, NC, USA

${ }^{2}$ Department of Chemistry, Morgan State University, Baltimore, MD, USA

${ }^{3}$ PrimBio Research Institute LLC, Exton, PA, USA

${ }^{4}$ Department of Pediatrics and Child Health, Howard University Medical School, Washington DC, USA

Email: *bhirendranath@ecsu.edu

How to cite this paper: Krauss, C., Aurelus, C., Johnston, K., Hedley, J., Banerjee, S., Wisniewski, S., Reaves, Q., Dia, K., Brown, S., Bartlet, V., Gavin, S., Cuffee, J., Banerjee, N., Rawat, K., Mandal, S., Abedin, Z., Ghosh, S. and Banerjee, H. (2022) A Study of Differential Gene Expression and Core Canonical Pathways Involved in Rhenium Ligand Treated Epithelial Mesenchymal Transition (EMT) Induced A549 Lung Cancer Cell Lines by INGENUITY Software System. Computational Molecular Bioscience, 12, 12-19.

https://doi.org/10.4236/cmb.2022.121002

Received: December 21, 2021

Accepted: March 4, 2022

Published: March 7, 2022

\begin{abstract}
Rhenium compounds have shown anti-cancer properties against many different types of cancer cell lines; however, the cellular signaling mechanisms involved in the cytotoxic properties of rhenium-based compounds were never deciphered or reported. In this manuscript, we report the results of an investigation done by RNA sequencing of rhenium treated A549 lung cancer cell lines along with an untreated vehicular control, analyzed by the Ingenuity Pathway Analysis (IPA) software system to decipher the core canonical pathways involved in rhenium induced cancer cell death. A549 EMT lung cancer cell lines were treated with rhenium ligand (Tricarbonylperrhenato(bathocuproine)rhenium(I), PR7) for seven days along with vehicular control. RNA was isolated from the treated and control cells and sequenced by a commercial company (PrimBio Corporation). The RNA sequencing data was analyzed by the INGNUITY software system and the core canonical pathways involved with differential gene expression were identified. Our report is showing that there are several cellular pathways involved in inducing cell death by rhenium-based compound PR7.
\end{abstract}

Keywords

Rhenium Compounds, Lung Cancer, Epithelial 
Copyright ( 2022 by author(s) and Scientific Research Publishing Inc. This work is licensed under the Creative Commons Attribution International License (CC BY 4.0). http://creativecommons.org/licenses/by/4.0/

\section{Introduction}

Rhenium based compounds have remained an interesting therapeutic strategy in the cancer biology field for decades. Rhenium ligands were reported to be toxic to cancerous cells while respiting the healthy cells; thus rhenium could potentially be a very effective drug for cancer treatments [1]. In this study, we tested the cytotoxicity of a rhenium ligand, Tricarbonylperrhenato(bathocuproine)rhenium(I) (PR7), against a GFP labeled vimentin gene knock in by CRISPR modified EMT model A549 lung cancer cell lines. These cell lines were created at ATCC (USA) to study therapeutic efficacy of anti-cancer compounds to prevent epithelial mesenchymal transition (EMT).

PR7 was previously reported to be bioactive against endometrial cancer cell lines [2]. In this study, we treated the vimentin knock in A549 lung cancer cell lines with PR7 after inducing EMT by TGF beta treatment and determined the cytotoxic effects of the drug by MTT assay technique. RNA was isolated from the PR7 treated EMT induced A549 cancer cells along with vehicular control (DMSO treated). The RNA was sequenced at a commercial RNA sequencing laboratory (PrimBio, LLC, USA). This sequencing data was then analyzed by the INGENUITY (IPA) software licensed from Qiagen Corporation, USA, to identify the potential effects of PR7 on gene expression and cellular signaling. In this manuscript, we report four core cellular canonical pathways that IPA reported and deciphered the differential gene expression due to the rhenium ligand treatment.

\section{Materials and Methods}

The rhenium ligand PR7 was synthesized as described previously [2]. The drug was dissolved in DMSO to form a solution.

The A549 EMT cell line was purchased from ATCC, USA and cultured in F-12K medium supplemented with FBS, Penicillin, and Streptomycin. The cells were kept in a $37^{\circ} \mathrm{C}$ incubator with $5 \% \mathrm{CO}_{2}$.

The MTT assay reagents were purchased from R\&D Systems, USA and the experiment was performed following the manufacturer's protocol after exposure to $1 \mu \mathrm{M}$ of PR7 for 48 hours. The results were read in a standard plate reader.

EMT was induced in the A549 cells by treating with $2.5 \mathrm{ng} / \mathrm{ml}$ TGF- $\beta$ for seven days along with $1 \mu \mathrm{M}$ PR7 and TGF- $\beta$ with $1 \mu \mathrm{M}$ DMSO treated cells only as vehicular control for the same time period.

RNA was isolated from TGF- $B$ and PR7 treated A549 cells and vehicular control cells after seven days treatment by using a total RNA isolation kit from Signosis Corporation, USA and then sent to PrimBio Corporation, USA for RNA sequencing. The data generated was then analyzed by the INGENUITY SYSTEM (IPA) software licensed from Qiagen Corporation, USA.

\section{Results}

Performing a MTT assay on A549 cells exposed to $1 \mu \mathrm{M}$ PR7 showed an increase in cell death compared to cells exposed to DMSO alone as a vehicular control 
(Figure 1). This proved that PR7 is bioactive in the A549 EMT cell line. We then analyzed by RNA sequencing the differential gene expression and by IPA analysis the core canonical pathways involved in PR7 treatment. Table 1 shows the major differentially expressed genes in response to PR7 treatment. Figure 2

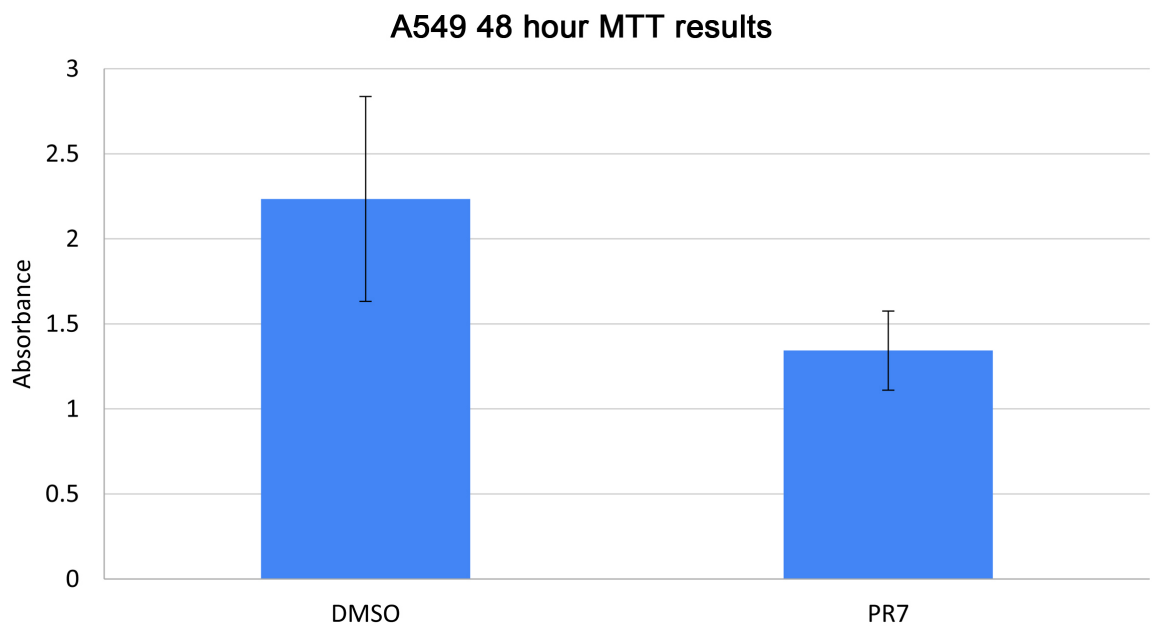

Figure 1. 48 hours MTT results of DMSO control vs $1 \mu$ M PR7 treated cells. A549 EMT Cells were grown in a 5\% $\mathrm{CO}_{2}$ incubator until confluent then treated with TGF beta and 1 $\mu \mathrm{M}$ of PR7 or an equal concentration of TGF beta and DMSO and returned to the incubator for 48 hours. Then MTT assay was performed, results showing increased cell death in PR7 treated cells.

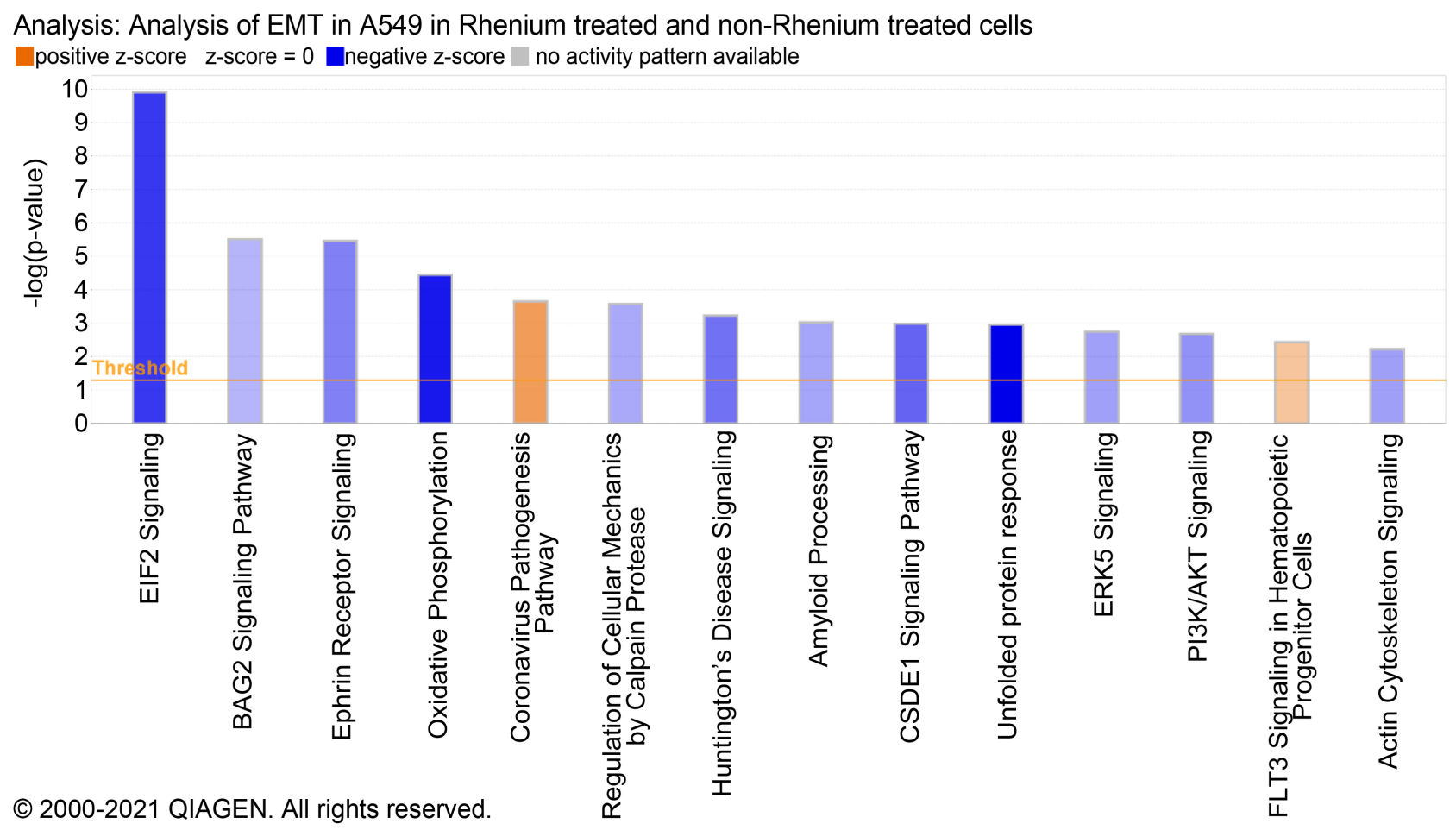

Figure 2. Core canonical pathways induced by PR7. This figure displays the core canonical pathways that PR7 treatment is likely to alter. Pathways shown in blue are anticipated to be downregulated, while an orange display shows the pathway to be likely upregulated. 
Table 1. Differentially expressed genes due to PR7 treatment with known role in lung cancer.

\begin{tabular}{|c|c|c|}
\hline Gene name & Expression Fold Change & Effect on lung cancer \\
\hline ACTG1 & -66.515 & $\begin{array}{l}\text { Found to be upregulated in small cell lung cancers. Overexpression has been } \\
\text { linked to higher metastatic potential in hepatocellular carcinoma. This suggests } \\
\text { that ACTG1 is likely assisting in cancer metastasis in lung cancers [14]. }\end{array}$ \\
\hline ENO1 & -177.945 & $\begin{array}{l}\text { Found to induce tumor growth and metastasis in vivo in lung } \\
\text { adenocarcinomas }[15]\end{array}$ \\
\hline FLNA & -67.865 & $\begin{array}{l}\text { High expression induces resistance to gefitinib, while lowering } \\
\text { expression restores sensitivity to gefitinib. Lower expression is } \\
\text { also able to induce apoptosis [3]. }\end{array}$ \\
\hline LARP4 & 25.148 & $\begin{array}{l}\text { Typically has a lower expression in non-small cell lung cancers including } \\
\text { A549. Higher expression could inhibit migration and invasion [4]. }\end{array}$ \\
\hline RPL19 & -115.264 & $\begin{array}{l}\text { Lowering RPL19 levels was found to inhibit the growth of lung } \\
\text { cancers that typically have an overexpression of RPL19. It is a } \\
\text { proposed target for immunotherapy [16]. }\end{array}$ \\
\hline RPS16 & -73.909 & Higher levels linked to lower survival rate in lung cancer patients [17]. \\
\hline RPS27A & -88.972 & $\begin{array}{l}\text { Direct transcriptional target of p53 that is highly overexpressed in lung cancer. } \\
\text { Appears to be a promising target for treatment [18]. }\end{array}$ \\
\hline TM4SF1 & -86.345 & $\begin{array}{l}\text { Upregulated in non-small cell lung cancers. Promotes cell proliferation, } \\
\text { migration, and invasion while also inhibiting apoptosis [19]. }\end{array}$ \\
\hline UBB & -84.321 & Overexpression leads to a lower survival rate in lung cancer patients [20]. \\
\hline YBX1 & -75.424 & High levels have been associated with poor prognosis in cancer patients [21]. \\
\hline
\end{tabular}

shows the different signaling pathways involved as deciphered by the IPA system due to PR7 treatment, in order to decipher the differential gene expression in A549 cells that were exposed to PR7, RNA isolated and sent for sequencing. There were two groups of A549 cells RNA that were sent for sequencing, both group of cells were exposed to TGF- $B$ to induce EMT, but only one group was exposed to PR7. The data generated from this sequencing was uploaded into the IPA software for a comparison analysis. This showed the differences in gene expression in the EMT induced A549 cells due to treatment with PR7. The data showed differences in over 90 core canonical pathways and calculated the changes in 9457 genes. Naturally all these pathways and genes were not relevant to lung cancer, so we selected four canonical pathways to examine and sorted the software's data to find the significant down and upregulated genes that could be associated with lung cancer.

\section{Discussions}

Our study showed that the rhenium ligand PR7 is cytotoxic to the A549 cancer cell line and induces differential gene expression. The drug treated CRISPR Cas9 modified vimentin-GFP knock in A549 lung cancer cell lines showed several downregulated and upregulated genes that are involved in cancer biogenesis pathways. We reported several such upregulated and downregulated genes rele- 
vant to lung cancer in Table 1 while Figure 2 shows the important core canonical pathways involved in PR7 treatment. Analyzing the downregulated genes, PR7 decreased expression of these oncogenes that helps in cancer progression. One of the genes found to be downregulated, FLNA, was reported to decrease drug resistance of lung cancer cells to gefitinib [3]. Gefitinib works by targeting the gene EGFR. This gene was also found to be downregulated due to the PR7 exposure, though not to the same degree as the other reported genes. Downregulation of EGFR was by an expression fold change of -3.334 due to PR7 exposure. This suggests that while PR7 does appear to show promising results on its own to treat lung cancer, it might also be able to be combined with gefitinib to increase efficacy. However, this combination was not able to be tested in our lab at this time, so the combinations efficacy cannot be stated certainly. Analyzing the upregulated genes, LARP4 upregulation was the only upregulated gene that was detrimental to cancer as its upregulation can inhibit migration and invasion [4]. The relevancy of the differential expression of these up and down regulated genes should be further investigated.

A brief discussion of the core canonical pathways that were identified by the IPA software system is described as follows.

The BAG2 signaling pathway was downregulated due to treatment with the PR7. This could delay tumor development due to the P53 downregulation at the end of the pathway. While P53 is a tumor suppressor gene, it is the most commonly mutated gene in cancers [5]. This mutation can occur due to BAG2 promoting the accumulation of mutated P53 [5]. When examining the molecules function of EIF2 signaling, which was the pathway with the best p-value, this pathway has some publications linking it to cancer. Inhibiting the EIF2 signaling pathway has been linked to a reduction in tumor growth in gastric cancers [6]. One study identified EIF2ß as a potential therapeutic target for non-small cell lung cancers [7]. Looking into the EIF2 signaling pathway more thoroughly in IPA showed that in the treated A549 cells, EIF2ß was downregulated.

Oxidative phosphorylation is typically shifted away from in cancers with most cancers favoring glycolysis as per the Warburg effect [8]. However, studies are showing that lung cancers do require oxidative phosphorylation to develop [8] [9]. Lung cancer cells with SMARCA4 mutations appear to be sensitive to inhibiting oxidative phosphorylation [9].

The ephrin signaling pathway is typically overexpressed in a variety of tumors. This promotes tumorigenesis, metastasis, and cancer stem cell regeneration [10]. Studies have identified this pathway as a target for drug development. PR7 was predicted to inhibit this pathway. A possible reason for this inhibition could be the downregulation of SHC. SHC binds with EPHA2, which is known to regulate tumor growth, migration, and invasiveness [11] [12]. However, in this same pathway, STAT3 was found to be upregulated which can promote metastasis [13]. Thus, our study shows the application of computational analysis by the IPA Software of molecular data obtained from RNA Sequencing and deciphering 
along with differential gene expression studies, analysis of the cellular core canonical pathways involved in potential anticancer therapeutic properties of the novel Rhenium ligand PR7.

\section{Acknowledgements}

This research is supported by NIH-NIGMS T34 GM 100831-09 awarded to Dr. H. Banerjee at the Elizabeth City State University campus of the University of North Carolina, USA.

\section{Conflicts of Interest}

The authors declare no conflicts of interest regarding the publication of this paper.

\section{References}

[1] Banerjee, H.N., Boston, A., Barfield, A., Stevenson, M., Sarkar, F.H., Giri, D., Winstead, A., Krause, J.A. and Mandal, S.K. (2016) A Study of the Effects of Novel Rhenium Compounds on Pancreatic and Prostate Cancer Cell Lines. International Journal of Scientific Research, 5, Article ID: 10501.

[2] Krauss, C., Aurelius, C., Johnston, K., Bartlette, V., Gavin, S., Cuffee, J., Banerjee, S., Wiseniewsky, S., Mandal, S. and Banerjee, H.N. (2020) An Investigation to Study the Role of Novel Rhenium Compounds on Endometrial Uterine Cancer Cell Lines. Journal of Cancer Research Updates, 9, 102-106. https://doi.org/10.30683/1929-2279.2020.09.12

[3] Cheng, L. and Tong, Q. (2021) Interaction of FLNA and ANXA2 Promotes Gefitinib Resistance by Activating the Wnt Pathway in Non-Small-Cell Lung Cancer. Molecular and Cellular Biochemistry, 476, 3563-3575. https://doi.org/10.1007/s11010-021-04179-1

[4] Shi, J.Q., Wang, B., Cao, X.Q., Wang, Y.X., Cheng, X., Jia, C.L., Wen, T., Luo, B.J. and Liu, Z.D. (2020) Circular RNA_LARP4 Inhibits the Progression of Non-Small-Cell Lung Cancer by Regulating the Expression of SMAD7. European Review for Medical and Pharmacological Sciences, 24, 1863-1869. https://doi.org/10.26355/eurrev 20200220364

[5] Yue, X., Zhao, Y., Liu, J., Zhang, C., Yu, H., Wang, J., Zheng, T., Liu, L., Li, J., Feng, Z. and Hu, W. (2015) BAG2 Promotes Tumorigenesis through Enhancing Mutant p53 Protein Levels and Function. eLife, 4, Article ID: e08401. https://doi.org/10.7554/eLife.08401

[6] Han, B., Yang, Y., Chen, J., He, X., Lyu, N. and Yan, R. (2019) PRSS23 Knockdown Inhibits Gastric Tumorigenesis through EIF2 Signaling. Pharmacological Research, 142, 50-57. https://doi.org/10.1016/j.phrs.2019.02.008

[7] Tanaka, I., Sato, M., Kato, T., Goto, D., Kakumu, T., Miyazawa, A., Yogo, N., Hase, T., Morise, M., Sekido, Y., Girard, L., Minna, J.D., Byers, L.A., Heymach, J.V., Coombes, K.R., Kondo, M. and Hasegawa, Y. (2018) eIF2 3 , a Subunit of Translation-Initiation Factor EIF2, Is a Potential Therapeutic Target for Non-Small Cell Lung Cancer. Cancer Science, 109, 1843-1852. https://doi.org/10.1111/cas.13602

[8] Kalainayakan, S.P., FitzGerald, K.E., Konduri, P.C., Vidal, C. and Zhang, L. (2018) Essential Roles of Mitochondrial and Heme Function in Lung Cancer Bioenergetics and Tumorigenesis. Cell \& Bioscience, 8, Article No. 56.

https://doi.org/10.1186/s13578-018-0257-8 
[9] Lissanu Deribe, Y., Sun, Y., Terranova, C., Khan, F., Martinez-Ledesma, J., Gay, J., Gao, G., Mullinax, R.A., Khor, T., Feng, N., Lin, Y.H., Wu, C.C., Reyes, C., Peng, Q., Robinson, F., Inoue, A., Kochat, V., Liu, C.G., Asara, J.M., Moran, C., et al. (2018) Mutations in the SWI/SNF Complex Induce a Targetable Dependence on Oxidative Phosphorylation in Lung Cancer. Nature Medicine, 24, 1047-1057. https://doi.org/10.1038/s41591-018-0019-5

[10] Lodola, A., Giorgio, C., Incerti, M., Zanotti, I. and Tognolini, M. (2017) Targeting Eph/Ephrin System in Cancer Therapy. European Journal of Medicinal Chemistry, 142, 152-162. https://doi.org/10.1016/j.ejmech.2017.07.029

[11] Bai, H., Duan, J., Li, C., Xie, W., Fang, W., Xu, Y., Wang, G., Wan, R., Sun, J., Xu, J., Wang, X., Fei, K., Zhao, Z., Cai, S., Zhang, L., Wang, J. and Wang, Z. (2020) EPHA Mutation as a Predictor of Immunotherapeutic Efficacy in Lung Adenocarcinoma. Journal for Immunotherapy of Cancer, 8, Article ID: e001315. https://doi.org/10.1136/jitc-2020-001315

[12] Pratt, R.L. and Kinch, M.S. (2002) Activation of the EphA2 Tyrosine Kinase Stimulates the MAP/ERK Kinase Signaling Cascade. Oncogene, 21, 7690-7699. https://doi.org/10.1038/sj.onc.1205758

[13] Zhang, X., Sai, B., Wang, F., Wang, L., Wang, Y., Zheng, L., Li, G., Tang, J. and Xiang, J. (2019) Hypoxic BMSC-Derived Exosomal miRNAs Promote Metastasis of Lung Cancer Cells via STAT3-Induced EMT. Molecular Cancer, 18, Article No. 40. https://doi.org/10.1186/s12943-019-0959-5

[14] Liu, J., Zhong, X., Li, J., Liu, B., Guo, S., Chen, J., Tan, Q., Wang, Q., Ma, W., Wu, Z., Wang, H., Hou, M., Zhang, H.T. and Zhou, Q. (2012) Screening and Identification of Lung Cancer Metastasis-Related Genes by Suppression Subtractive Hybridization. Thoracic Cancer, 3, 207-216. https://doi.org/10.1111/j.1759-7714.2011.00092.x

[15] Zhou, J., Zhang, S., Chen, Z., He, Z., Xu, Y. and Li, Z. (2019) CircRNA-ENO1 Promoted Glycolysis and Tumor Progression in Lung Adenocarcinoma through Upregulating Its Host Gene ENO1. Cell Death \& Disease, 10, Article No. 885. https://doi.org/10.1038/s41419-019-2127-7

[16] Kuroda, K., Takenoyama, M., Baba, T., Shigematsu, Y., Shiota, H., Ichiki, Y., Yasuda, M., Uramoto, H., Hanagiri, T. and Yasumoto, K. (2010) Identification of Ribosomal Protein L19 as a Novel Tumor Antigen Recognized by Autologous Cytotoxic T Lymphocytes in Lung Adenocarcinoma. Cancer Science, 101, 46-53. https://doi.org/10.1111/j.1349-7006.2009.01351.x

[17] Dai, D., Shi, R., Han, S., Jin, H. and Wang, X. (2020) Weighted Gene Coexpression Network Analysis Identifies Hub Genes Related to KRAS Mutant Lung Adenocarcinoma. Medicine, 99, Article ID: e21478. https://doi.org/10.1097/MD.0000000000021478

[18] Kidokoro, Y., Sakabe, T., Haruki, T., Kadonaga, T., Nosaka, K., Nakamura, H. and Umekita, Y. (2020) Gene Expression Profiling by Targeted RNA Sequencing in Pathological Stage I Lung Adenocarcinoma with a Solid Component. Lung Cancer, 147, 56-63. https://doi.org/10.1016/j.lungcan.2020.06.035

[19] Fu, X.Y., Zhou, W.B. and Xu, J. (2020) TM4SF1 Facilitates Non-Small Cell Lung Cancer Progression through Regulating YAP-TEAD Pathway. European Review for Medical and Pharmacological Sciences, 24, 1829-1840.

[20] Deng, H., Huang, Y., Wang, L. and Chen, M. (2020) High Expression of $U B B, R A C 1$, and ITGB1 Predicts Worse Prognosis among Nonsmoking Patients with Lung Adenocarcinoma through Bioinformatics Analysis. BioMed Research International, 2020, Article ID: 2071593. https://doi.org/10.1155/2020/2071593 
[21] Zhang, Y., Huang, Y.X., Wang, D.L., Yang, B., Yan, H.Y., Lin, L.H., Li, Y., Chen, J., Xie, L.M., Huang, Y.S., Liao, J.Y., Hu, K.S., He, J.H., Saw, P.E., Xu, X. and Yin, D. (2020) LncRNA DSCAM-AS1 Interacts with YBX1 to Promote Cancer Progression by Forming a Positive Feedback Loop That Activates FOXA1 Transcription Network. Theranostics, 10, 10823-10837. https://doi.org/10.7150/thno.47830 